\title{
ANÁLISE DA VARIAÇÃO DOS ELEMENTOS DO CAMPO DE GRAVIDADE NA REGIÃO DO AQÜÍFERO GUARANI A PARTIR DOS DADOS GRACE
}

\author{
Everton Pereira Bomfim e Eder Cassola Molina \\ Recebido em 25 outubro, 2007 / Aceito em 29 janeiro, 2009 \\ Received on October 25, 2007 / Accepted on January 29, 2009
}

\begin{abstract}
This work estimates the variation of the gravity field elements in a period of two years and three months - April 2002 to July 2004 - using the GRACE mission data in the Guarani aquifer region; these changes are the sum of all the gravity changes that are caused by variations from mass distribution of the Earth's surface and by the deformation of the solid Earth in response to these mass variations. The solutions have been calculated only from the disturbances of the satellite orbits and do not depend on the gravity data available in the oceanic and continental areas. The calculations of the Earth's gravity field monthly solutions by using the GRACE mission data, considering the spherical harmonics, i.e., the Stokes coefficients, for the global gravity field, up to degree and order 150 (February/2003 to July/2004) and 120 (April, August and November 2002), have been transformed in geoidal height and free-air anomaly grids, with the respective associated errors. A linear 3-D inversion using the residual geoid height supplied the three dimensional density distribution, showing that the use of the GRACE data in investigation of the water mass variability in Guarani aquifer is feasible. A Bouguer anomaly profile, calculated by using the GRACE free-air anomaly and a digital topographic model data, was used in order to estimate the water mass variation in the Guarani aquifer region.
\end{abstract}

Keywords: Guarani aquifer, geopotential, geoid, GRACE satellites, spherical harmonic, linear inversion.

RESUMO. Este trabalho visa estimar e analisar a variação dos elementos do campo de gravidade na região do Aqüífero Guarani em um período de dois anos e três meses - abril de 2002 a julho de 2004 - usando os dados da missão GRACE. Esta variação engloba todas as mudanças de gravidade que são causadas por variações na distribuição de massa da superfície da Terra e pela deformação da Terra Sólida em resposta a estas variações de massa, estimadas pelos coeficientes de Stokes fornecidos pela missão GRACE. As soluções foram obtidas somente a partir de perturbações de órbita de satélites independentemente de dados de gravidade na superfície oceânica e continental. As soluções mensais do campo gravitacional da Terra fornecidas pela missão GRACE, considerando o grau e a ordem máximos de expansão dos elementos do campo de gravidade em harmônicos esféricos de 150 (fevereiro de 2003 a julho de 2004) e 120 (abril, agosto e novembro de 2002), foram processadas e forneceram valores de altura geoidal e anomalia ar-livre para cada conjunto destas soluções mensais, juntamente com os erros associados. Uma inversão linear 3-D da altura geoidal residual foi realizada para obter a distribuição de densidades tridimensional associada a estas variações, verificando-se assim a viabilidade do uso dos dados do GRACE na investigação da variação das massas de água no Aqüífero Guarani. A partir da anomalia ar-livre e dos dados de um modelo topográfico digital da região obteve-se também a anomalia Bouguer em um perfil representativo do aqüífero, a fim de estimar a correspondente variação da massa de água na região analisada.

Palavras-chave: Aqǘfero Guarani, geopotencial, geóide, satélites GRACE, harmônicos esféricos, inversão linear. 


\section{INTRODUÇÃO}

\section{Aqüífero Guarani}

0 Aqǘfero Guarani é a principal reserva de água doce da América do Sul e um dos maiores sistemas aqüíferos do mundo. Segundo a CETESB (2001) está localizado na região centro-leste da América do Sul, entre $12^{\circ}$ e $35^{\circ}$ de latitude sul e entre $47^{\circ}$ e $65^{\circ}$ de longitude oeste e ocupa uma área total de 1,2 milhões de $\mathrm{km}^{2}$ na Bacia do Paraná e parte da Bacia do Chaco-Paraná. Estende-se pelo Brasil $\left(840.000 \mathrm{~km}^{2}\right)$, Paraguai $\left(58.500 \mathrm{~km}^{2}\right)$, Uruguai $\left(58.500 \mathrm{~km}^{2}\right)$ e Argentina, $\left(255.000 \mathrm{~km}^{2}\right)$, abrangendo uma área equivalente à soma dos territórios da Inglaterra, França e Espanha. Sua maior ocorrência se dá em território brasileiro (2/3 da área total) abrangendo os Estados de Goiás, Mato Grosso do Sul, Minas Gerais, São Paulo, Paraná, Santa Catarina e Rio Grande do Sul (CETESB, 2004).

0 Aqüífero Guarani é formado pelo preenchimento de água nos espaços das rochas (poros e fissuras), convencionalmente denominadas Guarani. As rochas do Guarani se constituem de camadas arenosas depositadas no período de 944 e 245 milhões de anos (Rocha, 1997). A espessura das camadas de água que compõem o Aqüífero Guarani varia de 50 a 800 metros, estando situadas em profundidades que podem atingir até 1800 metros. As camadas que constitui o Aqǘfero Guarani têm arquitetura arqueada para baixo como resultado da pressão das rochas sobrejacentes, como os espessos derrames de lavas basálticas oriundos da ativação de falhas, arcos regionais e soerguimento de bordas, ocorridos na Bacia Sedimentar do Paraná.

As formações geológicas que compõem o Sistema Aqüífero Guarani congregam sedimentos fluvio-lacustres do período Triássico (245-208 milhões de anos): Formações Pirambóia e Rosário do Sul, no Brasil, e Buena Vista no Uruguai; sedimentos eólicos desérticos do período Jurássico (208-144 milhões de anos): Formações Botucatu, no Brasil; Misiones, no Paraguai; e Tacuarembó no Uruguai e Argentina (Rocha, 1997).

\section{A missão GRACE}

A missão GRACE (Gravity Recovery and Climate Experiment) consiste de dois satélites artificiais idênticos que foram colocados na mesma órbita polar a aproximadamente 500 quilômetros de altitude e separados a 220 quilômetros um do outro. À medida que os dois satélites orbitam em torno da Terra, as regiões do planeta que possuem uma gravidade ligeiramente mais forte afetarão primeiro o satélite líder, que por sua vez será ligeiramente afastado do outro que o acompanha. As distâncias de separação entre eles podem ser monitoradas com extraordinária precisão: os dois satélites são capazes de aferir uma mudança na sua separação equivalente a um micrômetro.

Por meio de medidas constantes das mudanças na distância entre os dois satélites combinados com os dados das medidas de posição do sistema de posicionamento global GPS, e aferições dos acelerômetros a bordo de cada satélite e velocidades precisas é possível determinar variações muito pequenas no campo de gravidade da Terra. A missão GRACE usa um sistema de microondas ressonantes da banda $\mathrm{K}$ que aferem as velocidades e distâncias entre os dois satélites devido às mudanças do campo gravitacional da Terra que estão correlacionadas às mudanças na massa (topografia) e irregularidades nas distribuições de densidades de massa, onde o movimento orbital de cada satélite é alterado. Esta mudança no movimento orbital que causa mudanças nas distâncias entre os satélites são aferidas pelo instrumento de banda K. 0 sistema ressonante é extremamente sensível, o que permite esta determinação com a grande precisão necessária para determinar o campo gravitacional da Terra.

No passado, a técnica de Satellite Laser Ranging (SLR) fora usada para determinar mudanças sazonais de longo comprimento de onda do campo de gravidade devidas às transferências de massas entre a atmosfera, oceano, e fontes de águas continentais. Estas medidas eram limitadas em resolução por causa da distribuição geográfica dos dados coletados e pelas elevadas altitudes dos satélites. A missão GRACE foi implementada para prever medidas globais desse mesmo fenômeno, mas com meIhor resolução espacial e precisão que 0 anterior.

A magnitude de erros na estimativa dos coeficientes dos modelos do GRACE varia mês a mês por causa da combinação de diversos fatores, incluindo a cobertura espacial dos dados coletados, a cobertura temporal (i.e., dias com falta de dados) e 0 intervalo de tempo investigado (Tapley et al., 2004). No entanto, a acurácia dos dados obtidos da missão GRACE é estimada na forma da amplitude do grau do erro do geóide esperado, estimativas esperadas da altura geoidal como uma função do grau, 0 qual assume-se que as incertezas nas estimativas dos coeficientes dos harmônicos esféricos $C_{l m}$ e $S_{l m}$ fornecidos dependem do grau e não da ordem (isto é, o erro no geóide depende do comprimento de onda, mas não da posição espacial, ou seja, na média, as medidas da missão GRACE determinam igualmente bem o geóide em todas as regiões do globo terrestre) (Wahr et al., 1998). 0 erro na estimativa dos coeficientes é da ordem de $10^{-3}$ metros (0.32 a 14.6 milímetros) para grau e ordem 70.

0 erro nos dados obtidos da missão GRACE foi analisado na forma do grau de amplitude (variância do grau), e foi constatado, 
conforme mostra a Figura 1, que os erros aumentam com 0 aumento do grau I, isto é, com a diminuição da escala espacial. Considera-se que 0 erro total é determinado pela média de 16 soluções do campo de gravidade GRACE de grau e ordem 150, (soluções de fevereiro de 2003 a julho de 2004) e 120 (abril, agosto e novembro de 2002), ou seja, a média do erro formal (erro de calibração) fornecido pelo GRACE para cada conjunto de solução fornecido pelos satélites GRACE com relação à escala espacial $(20.000 \mathrm{~km} / \mathrm{l})$. As soluções utilizadas neste trabalho foram os modelos do campo de gravidade da Terra completo para grau e ordem EIGEN-GRACE01S e EIGEN-GRACE02S (European Improved Gravity model of the Earth by New techniques solely from GRACE Satellite data) para grau e ordem 120 e 150 respectivamente (Reigber et al., 2003 e 2005). Conforme 0 trabalho de Wahr et al. (1998), assume-se que as soluções do campo de gravidade dos dados obtidos da missão GRACE, os quais são os coeficientes dos harmônicos esféricos $C_{l m}$ e $S_{l m}$ para grau e ordem máximos iguais a 120 ou 150, são afetadas por erros aleatórios e que no cálculo da variância do grau não estão computados os erros devidos aos coeficientes $C_{20}$ e nem os erros para os coeficientes de grau 0 e $1(l=0$ e $l=1)$.
Nesta figura observa-se um erro mínimo de aproximadamente $0.32 \mathrm{~mm}$ para grau e ordem 10, sendo o erro para grau e ordem 150 de aproximadamente $444 \mathrm{~mm}$.

Os resultados das variações temporais de gravidade medidas pelo GRACE são um importante vínculo adicional na estimativa de modelos hidrológicos, pois representam a integração do efeito da massa de água sobre uma coluna vertical; sendo assim, não é possível distinguir entre as diferentes fontes que causaram a variação no campo de gravidade (Tapley et al., 2004). Numericamente, a separação de cada componente (a contribuição de cada fluido) é um problema que requer informação suplementar, como por exemplo, outros tipos de dados de satélite, medidas in situ, ou valores previstos por variáveis hidrológicas baseados em modelos de clima global (Ramillien et al., 2004).

Deve-se notar que a conformidade perfeita dos resultados do GRACE com os modelos de hidrologia de solo não é necessariamente esperada, mesmo onde estes estiverem disponíveis com relativa precisão. Por exemplo, é comum que modelos globais de hidrologia continental, como 0 GLDAS (Rodell et al., 2004), não incluam no modelo os efeitos de fluxo de água profunda,
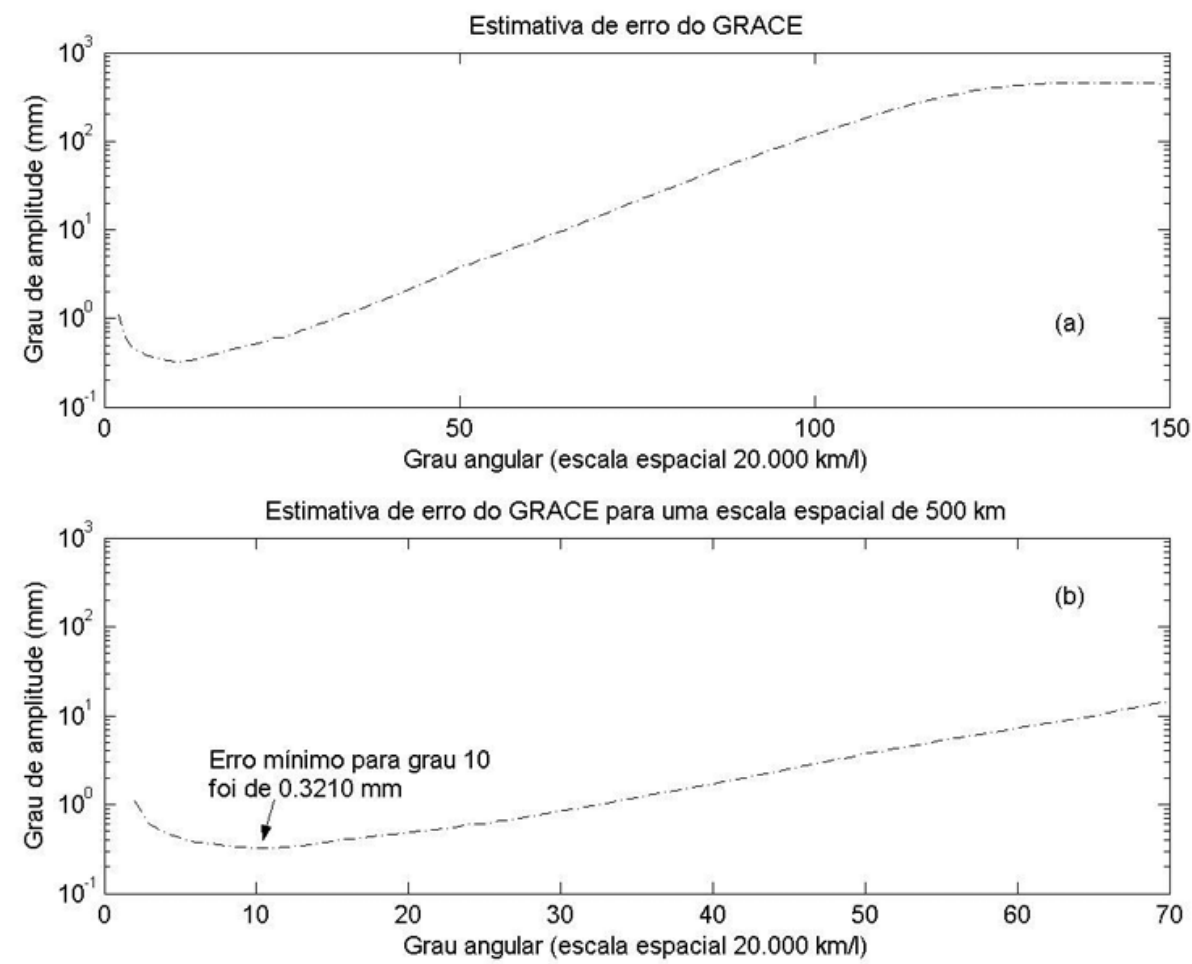

Figura 1 - Amplitude do erro para a componente de variação do geóide: estimativas da amplitude dos erros GRACE calibrados em unidades de altura geoidal. (a) Erro para os dados do GRACE com $L=M=150$ (grau e ordem máximos). (b) Amplitude do erro para uma resolução espacial de aproximadamente 286 km (grau e ordem 70). 
persistência de neve, ou de grandes variabilidades no continente, como no caso da Antártica.

\section{Equações relacionando a distribuição superficial de massa com a gravidade}

Este trabalho utiliza estimativas da variabilidade da distribuição superficial de massa para obter a resposta da gravidade e aqui foram utilizados os dados de gravidade dos satélites GRACE (dados de soluções de geóides mensais) para estimar essa variabilidade da distribuição superficial de massa em subsuperfície (Wahr et al., 1998). Para tanto, utilizou-se equações que relacionam as mudanças na distribuição superficial de massa com a gravidade.

A altura geoidal descreve 0 campo de gravidade global da Terra, e é geralmente descrita como a soma dos harmônicos esféricos (Heiskanen \& Moritz, 1967)

$$
\begin{aligned}
N(\theta, \phi)= & a \sum_{l=0}^{\infty} \sum_{m=0}^{l} \tilde{P}_{l m}(\cos \theta) \\
& \left(C_{l m} \cos (m \phi)+S_{l m} \sin (m \phi)\right),
\end{aligned}
$$

onde $a$ é o raio da Terra, $\theta$ e $\phi$ são a co-latitude e a longitude respectivamente, $C_{l m}$ e $S_{l m}$ são coeficientes de Stokes adimensionais, e $\tilde{P}_{l m}$ são os polinômios de Legendre associados plenamente normalizados

$$
\begin{gathered}
\tilde{P}_{l m}(x) \sqrt{\left(2-\delta_{m 0}\right)(2 l+1) \frac{(1-m) !}{(1+m) !}} \\
\frac{\left(1-x^{2}\right)^{\frac{m}{2}}}{2^{l} l !} \frac{d^{l+m}}{d x^{l+m}}\left(x^{2}-1\right)^{l} .
\end{gathered}
$$

Os dados da missão GRACE, soluções do campo de gravidade (geóide) definidas simplesmente por soluções mensais dos coeficientes do geopotencial, consistem tipicamente de valores para as variáveis dos coeficientes dos harmônicos esféricos $C_{l m}$ e $S_{l m}$ mensais, isto é, estes coeficientes são calculados a partir de um conjunto de dados abrangendo um período de coleta de aproximadamente 30 dias pelos satélites GRACE e vão até 0 grau e ordem máximos 150 na expansão dos harmônicos esféricos para as estimativas mensais do GRACE aqui obtidas (Reigber et al., 2005), onde estas soluções mensais de fevereiro de 2003 a julho de 2004 vêm acompanhadas de um arquivo de erros calibrados (desvio padrão), isto é, raiz média quadrada (rms) predita pelo erro da altura geoidal baseado no desvio padrão da matriz de covariância. Entretanto, as soluções mensais do campo de gravidade fornecidas pela missão GRACE foram processadas para obter modelos de alturas geoidal mensais, ou seja, valores mensais para as variáveis $C_{l m}$ e $S_{l m}$ para grau e ordem máximos 120 e 150.

Supondo que haja uma variação temporal no geóide $(\Delta N)$, pode-se então imaginá-la como representando uma mudança qualquer em $N$ de um tempo $t 1$ para um tempo $t 2$, ou como a diferença entre $N$ em um tempo $t 1$ e uma média de $N$ calculada para um período de tempo $d t$, ou como alguma outra representação de uma mudança em $N$. De qualquer forma, esta variação $\Delta N$ pode ser representada em termos de mudanças nos coeficientes dos harmônicos esféricos $\Delta C_{l m}$ e $\Delta S_{l m}$, pela equação

$$
\begin{aligned}
\Delta N(\theta, \phi)= & a \sum_{l=0}^{\infty} \sum_{m=0}^{l} \tilde{P}_{l m}(\cos \theta) \\
& \left(\Delta C_{l m} \cos (m \phi)+\Delta S_{l m} \sin (m \phi)\right) .
\end{aligned}
$$

A variação nesses coeficientes é causada pela redistribuição de densidade superficial $(\Delta \sigma)$, que é definida como massa dividida pela área.

A variação nos coeficientes dos harmônicos esféricos do geóide pode ser dividida em duas partes. A primeira parte descreve a contribuição ao geóide devido à atração gravitacional da distribuição superficial de massa. Por sua vez, essa distribuição superficial de massa é tida como uma carga (peso) que deforma elasticamente a Terra Sólida adjacente causando assim uma contribuição adicional ao geóide, representada pela segunda parte:

$$
\left\{\begin{array}{c}
\Delta C_{l m} \\
\Delta S_{l m}
\end{array}\right\}=\left\{\begin{array}{l}
\Delta C_{l m} \\
\Delta S_{l m}
\end{array}\right\}_{\text {surf mass }}+\left\{\begin{array}{c}
\Delta C_{l m} \\
\Delta S_{l m}
\end{array}\right\}_{\text {solid } E} .
$$

Isto resulta na Eq. (5), que inclui as duas contribuições:

$$
\begin{gathered}
\left\{\begin{array}{c}
\Delta C_{l m} \\
\Delta S_{l m}
\end{array}\right\}=\frac{3\left(1+k_{l}\right)}{4 \pi a \rho_{a v e}(2 l+1)} \\
\int \Delta \sigma(\theta, \phi) \tilde{P}_{l m}(\cos \theta)\left\{\begin{array}{c}
\cos (m \phi) \\
\sin (m \phi)
\end{array}\right\} \sin \theta d \theta d \phi .
\end{gathered}
$$

Nesta equação, $k_{l}$ é número de Love elástico de grau $l$, $\rho_{a v e}$ é densidade média da Terra e $\Delta \sigma$ é a mudança na densidade de superfície causada pelas variações em questão. Sendo 0 campo de gravidade da Terra variável no tempo e espaço que por sua vez define um geóide, onde este origina principalmente pelas irregularidades na distribuição de massas próximas à superfície da Terra, as quais consistem de uma parte estática e uma parte variável no tempo (Wahr et al., 1998). A parte estática é principalmente devido às distribuições de massas que somente variam 
em uma Escala Geológica de Tempo, como continentes, montanhas e depressões na crosta. A parte variável no tempo ocorre devido aos processos como a redistribuição do armazenamento de água terrestre, marés oceânicas, mudanças atmosféricas e ressurgência pós-glacial, ou seja, o sinal hidrológico, por exemplo, a redistribuição do armazenamento de água terrestre, é incluído nos dados da gravidade obtidos pela missão GRACE. Portanto, considera-se o sinal hidrológico do GRACE como dados 'sintéticos', uma vez que foram estimativas indiretas dos dados hidrológicos. Este procedimento é chamado 'estimativa inversa dos dados do GRACE' (Gerrits, 2005). A Eq. (5) pode ser usada para obter irregularidades na distribuição de massas próximas à superfície da Terra.

Reescrevendo a Eq. (5), com $\rho_{w}$ sendo a densidade da água, tem-se a Eq. (6):

$$
\begin{gathered}
\Delta \sigma(\theta, \phi)= \\
\frac{a \rho_{\text {ave }}}{3} \sum_{l=0}^{\infty} \sum_{m=0}^{l} \tilde{P}_{l m}(\cos \theta) \frac{2 l+1}{1+k_{l}} \\
\left(\Delta C_{l m} \cos (m \phi)+\Delta S_{l m} \sin (m \phi)\right) .
\end{gathered}
$$

Dividindo $\Delta \sigma$ por $\rho_{w}$, a mudança na massa superficial obtida é expressa em espessura equivalente em água. Esta equação pode ser usada para calcular o sinal hidrológico dos dados medidos pelo sistema GRACE. Este método é referido como 'estimação direta da hidrologia' a partir dos dados do GRACE.

\section{Tratamento dos dados de gravidade dos satélites GRACE para uma análise em perfil}

Como uma maneira de avaliar a variação de massa de água no Aqüífero Guarani a partir dos dados do GRACE, as soluções mensais do campo de gravidade (em coeficientes do geopotencial truncados em grau e ordem 70 , uma vez que os dados da missão GRACE para 0 grau e ordem mais elevados possuem erros mais elevados, de onde foram calculadas as anomalias ar-livre mensais e, a partir destas anomalias e de um modelo topográfico digital calculado usando os dados do SRTM, foram obtidas estimativas mensais da anomalia Bouguer ao longo de um perfil representativo da região de estudo de aproximadamente $1.860 \mathrm{~km}$, representado na Figura 2 (perfil $A-A^{\prime}$ ).

\section{Estimativa da anomalia ar-livre, anomalia Bouguer e topografia em perfil}

Os valores da estimativa da anomalia Bouguer foram calculados pela expressão

$$
\Delta g_{B}=g-\gamma+C_{F}(h)+C_{B}(h),
$$

onde $0 \mathrm{~g}$ é a gravidade medida no ponto de observação, $\gamma$ é a gravidade normal calculada para a projeção do mesmo ponto no elipsóide de referência, que, segundo o GRS67 (Geodetic Reference System 1967), é expressa em mGal $\left(10^{-3} \mathrm{Gal}=10^{-5} \mathrm{~m} / \mathrm{s}^{2}\right)$. Neste trabalho, adotou-se como modelo global a Fórmula Internacional de Gravidade definida em 1967 pela International Union of Geodesy and Geophysics (IUGG) do International Association of Geodesy (IAG) (IAG, 1971):

$$
\begin{gathered}
\gamma=978031,85\left(1+0,005278895 \operatorname{sen}^{2} \varphi\right. \\
\left.+0,000023462 \operatorname{sen}^{4} \varphi\right),
\end{gathered}
$$

onde $\phi$ é a latitude do ponto de observação da gravidade $(g)$, $C_{F}(h)$ é a correção ar-livre, $C_{B}(h)$ é a correção Bouguer e $h$ é a altitude ortométrica do ponto de observação, em metros.

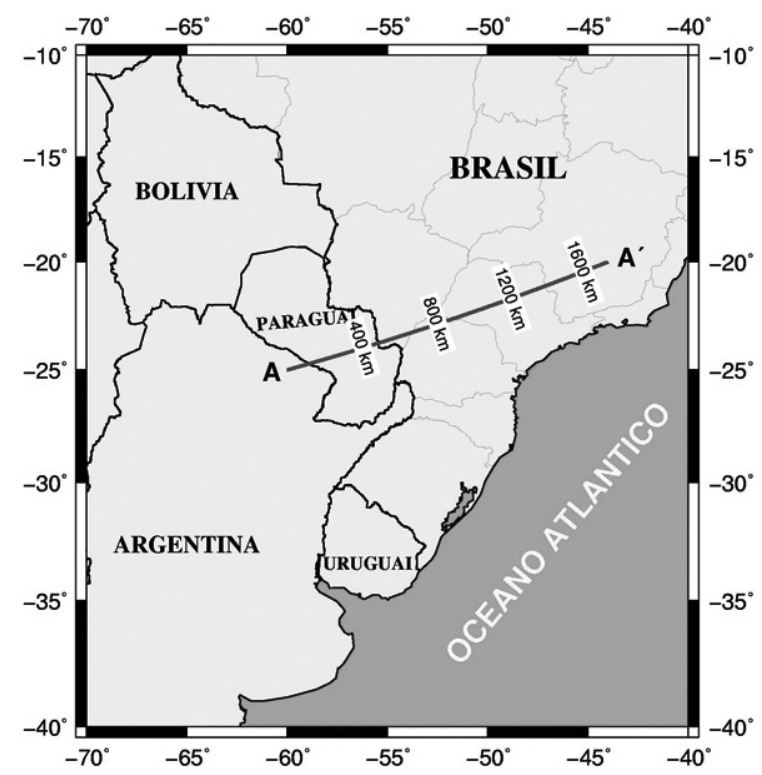

Figura 2 - Perfil A-A analisado para estimativa da variação da anomalia Bouguer.

A anomalia ar-livre pode ser obtida segundo a relação $\Delta g_{a}=g-\gamma+0,3086 h$.

No presente trabalho, a anomalia ar-livre foi calculada diretamente a partir dos coeficientes fornecidos pelo GRACE. Na Figura 3 estão representadas as anomalias ar-livre ao longo do perfil A-A' (Fig. 2), a cada dois meses consecutivos de dados do campo de gravidade da Terra coletados pelos satélites GRACE para uma análise das anomalias mês a mês. Não foram possiveis obter as anomalias para os meses de janeiro de 2003, janeiro de 2004 e junho de 2003, devidos à falta de dados coletados pelos satélites em quantidade e qualidade suficientes para gerar 0 campo de gravidade nestes períodos. As soluções do campo de 

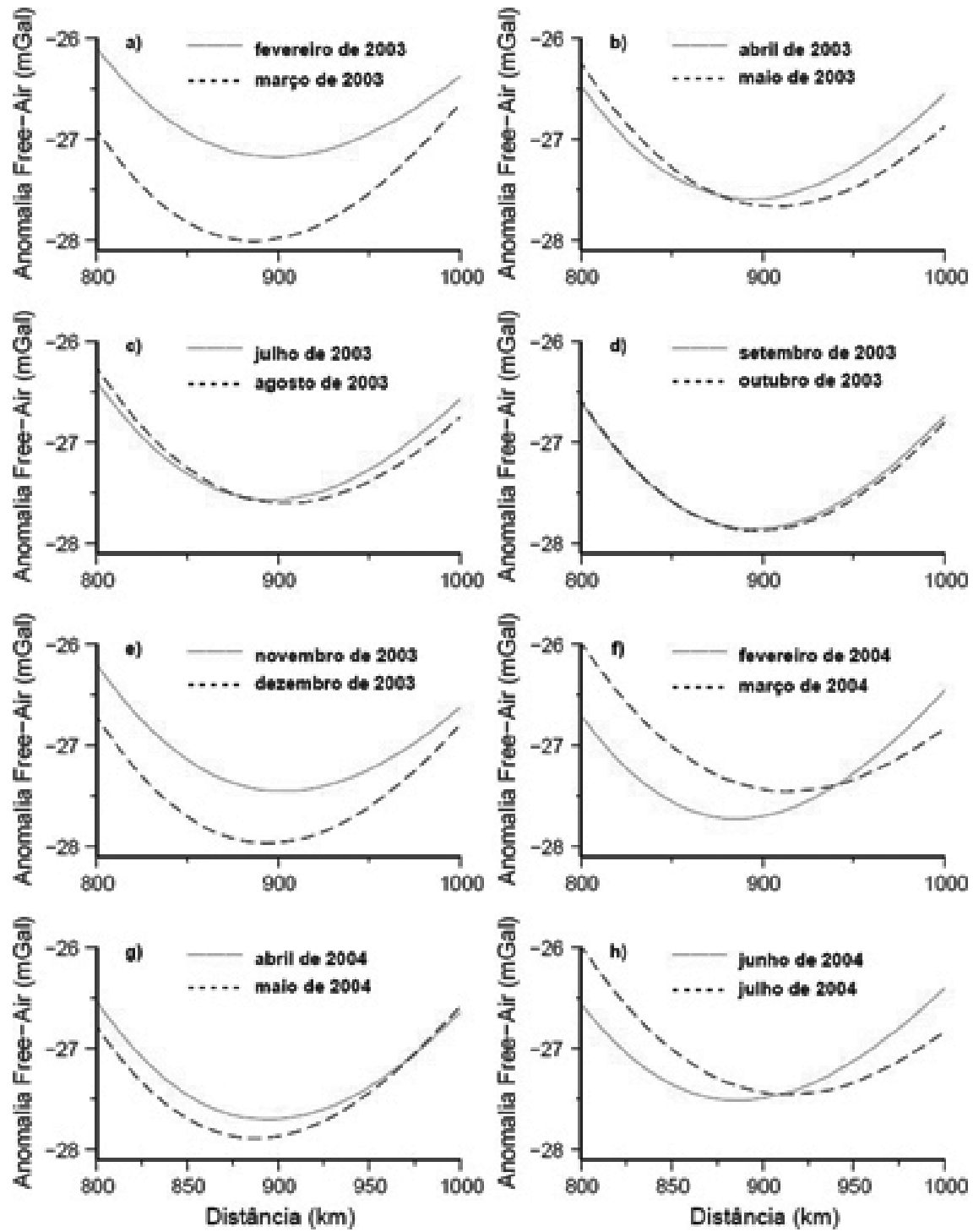

Figura 3 - Ampliação no trecho entre 800 e 1000 km no Perfil A-A' da anomalia ar-livre para cada solução mensal.

gravidade para os meses de abril, agosto e novembro de 2002 com grau e ordem máximos 120 também foram processados pelos mesmos procedimentos obtidos neste trabalho.

Observa-se que a anomalia ar-livre mantém um padrão similar mês a mês ao longo do perfil analisado, o que é razoável, uma vez que anomalia de longo comprimento de onda dessa região, gerada pelas características geológicas, não muda com tempo (no curto intervalo de tempo); porém, observando-se uma ampliação em determinado trecho do perfil, percebe-se uma pequena variação dessa grandeza de um mês em relação ao outro (Fig. 3), o que mostra que os dados obtidos da missão GRACE conseguem detectar variações temporais do campo de gravidade para este intervalo de tempo e de grandeza.
A estimativa mensal da anomalia Bouguer foi obtida utilizando-se a relação dada por (Heiskanen \& Moritz, 1967)

$$
\begin{aligned}
\Delta g_{B} & =g-\gamma+0,3086 h-2 \pi G \rho h \\
& =\Delta g_{a}-0,1119 h,
\end{aligned}
$$

onde $G=6,672 \times 10^{-11} \mathrm{~m}^{3} \mathrm{~kg}^{-1} \mathrm{~s}^{-2}$ é 0 valor da constante de gravitação universal e $\rho=2,67 \mathrm{~g} / \mathrm{cm}^{3}$ é 0 valor de densidade média da crosta terrestre normalmente utilizado para 0 cálculo da anomalia Bouguer. Para obter 0 valor de $h$ em cada ponto observado do perfil A-A' foi utilizado o modelo topográfico digital do Shuttle Radar Topography Mission (SRTM) (van Zyl, 2001), de forma a obter, juntamente com a anomalia ar-livre, a estimativa da anomalia Bouguer em perfil. Para a suavização 
topográfica, de maneira a se ter uma estimativa da anomalia Bouguer sem a introdução de componentes espúrias de curto comprimento de onda, foi aplicado o filtro gaussiano aos dados. Foram utilizadas várias resoluções de suavização gaussiana e 0 filtro gaussiano com um raio espacial médio (meio comprimento de onda) de $200 \mathrm{~km}$ foi 0 que melhor se ajustou aos dados topográficos, de forma a obter uma anomalia Bouguer média que mostra as mesmas características para longo comprimento de onda da anomalia Bouguer obtida com dados gravimétricos terrestres da região (Sá et al., 1993). A Figura 4 mostra a comparação da anomalia Bouguer estimada pelo GRACE com anomalia Bouguer obtida por Sá et al., op. cit.

A primeira vista, não se percebe variação significativa da estimativa da anomalia Bouguer mês a mês obtida dos satélites GRACE, considerando-se os longos e médios comprimentos de onda; os maiores valores observados estão a sudoeste do perfil e os valores menores estão na região nordeste (Fig. 4); porém, ampliando-se um trecho do perfil A-A' entre 850 e 950 km, podese perceber uma pequena variação mensal dessa estimativa, que aqui é atribuída à variação de massa de água, visto que as demais variáveis geológicas que são responsáveis pela variação do campo de gravidade permanecem constantes neste intervalo de tempo. Na mesma figura nota-se que estimativa da anomalia Bouguer obtida a partir de uma solução média, que possui 376 dias de coleta de dados da missão GRACE, com expansão dos elementos do campo de gravidade em harmônicos esféricos de grau e ordem máximos 150 do GRACE condiz com a anomalia Bouguer obtida dos dados da representação integrada do campo de gravidade no Brasil (Sá et al., 1993).

\section{Estimativa da variação da massa de água para justificar os valores de anomalia Bouguer}

A modelagem direta das estimativas da anomalia Bouguer em perfil se deu para estimar quantitativamente, utilizando o platô de Bouguer, a variação de um volume de água em uma área retangular de espessura h mês a mês, conforme mostra a Figura 6 . Este volume de água representa a quantidade total do fluido que gera o sinal gravimétrico observado, e não tem correspondência com nenhum cenário geológico, servindo apenas para quantificar a variação do volume total de fluido ao longo do tempo.

Uma vez obtidas as estimativas das anomalias Bouguer para cada solução mensal, removeu-se então uma média da estimativa Bouguer, valores para as variáveis dos coeficientes dos harmônicos esféricos $C_{l m}$ e $S_{l m}$ calculados a partir de um conjunto de dados abrangendo um período de coleta de aproximadamente 376 dias pelos satélites GRACE, de cada estimativa Bouguer mensal, buscando estimar volume de água necessário para justificar esta anomalia residual. As variações mensais das estimativas da anomalia Bouguer representadas na Figura 7 foram

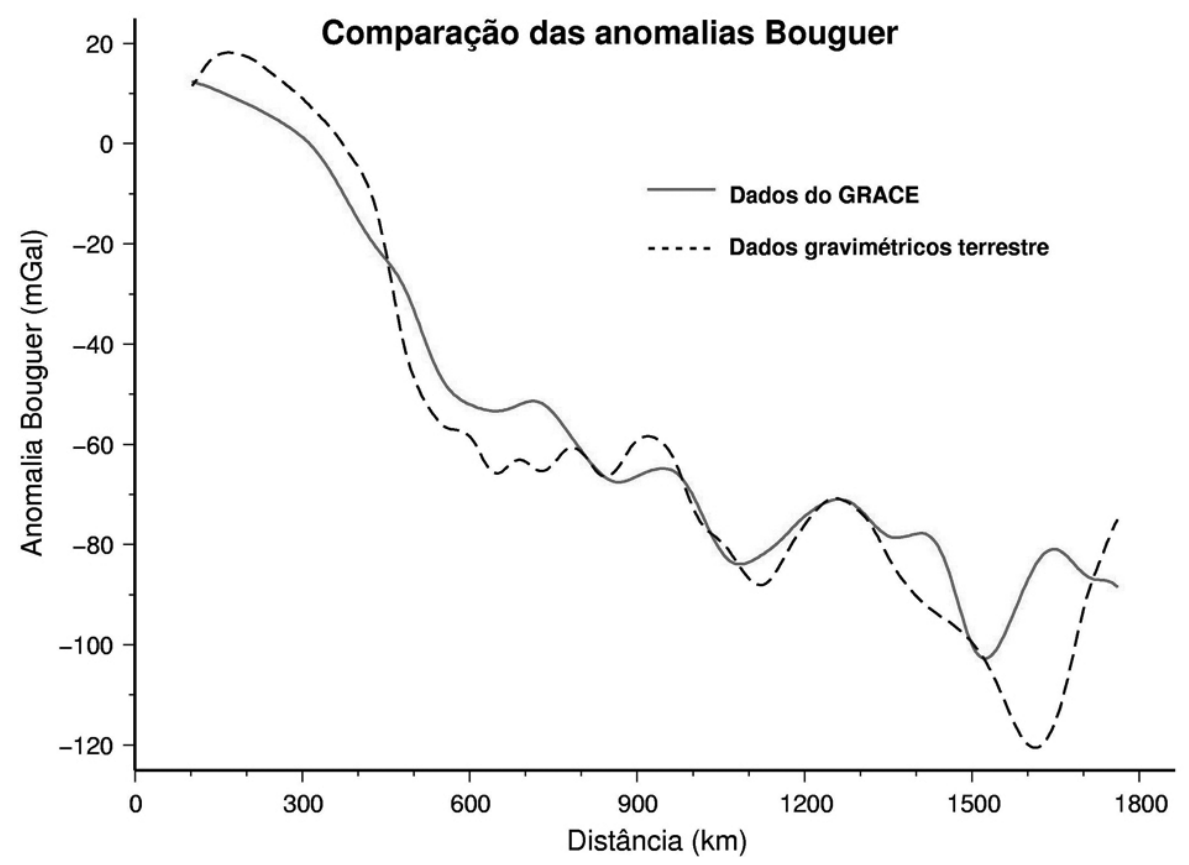

Figura 4 - Comparação da estimativa da anomalia Bouguer dos satélites GRACE com anomalia Bouguer estimada pelo levantamento gravimétrico. 

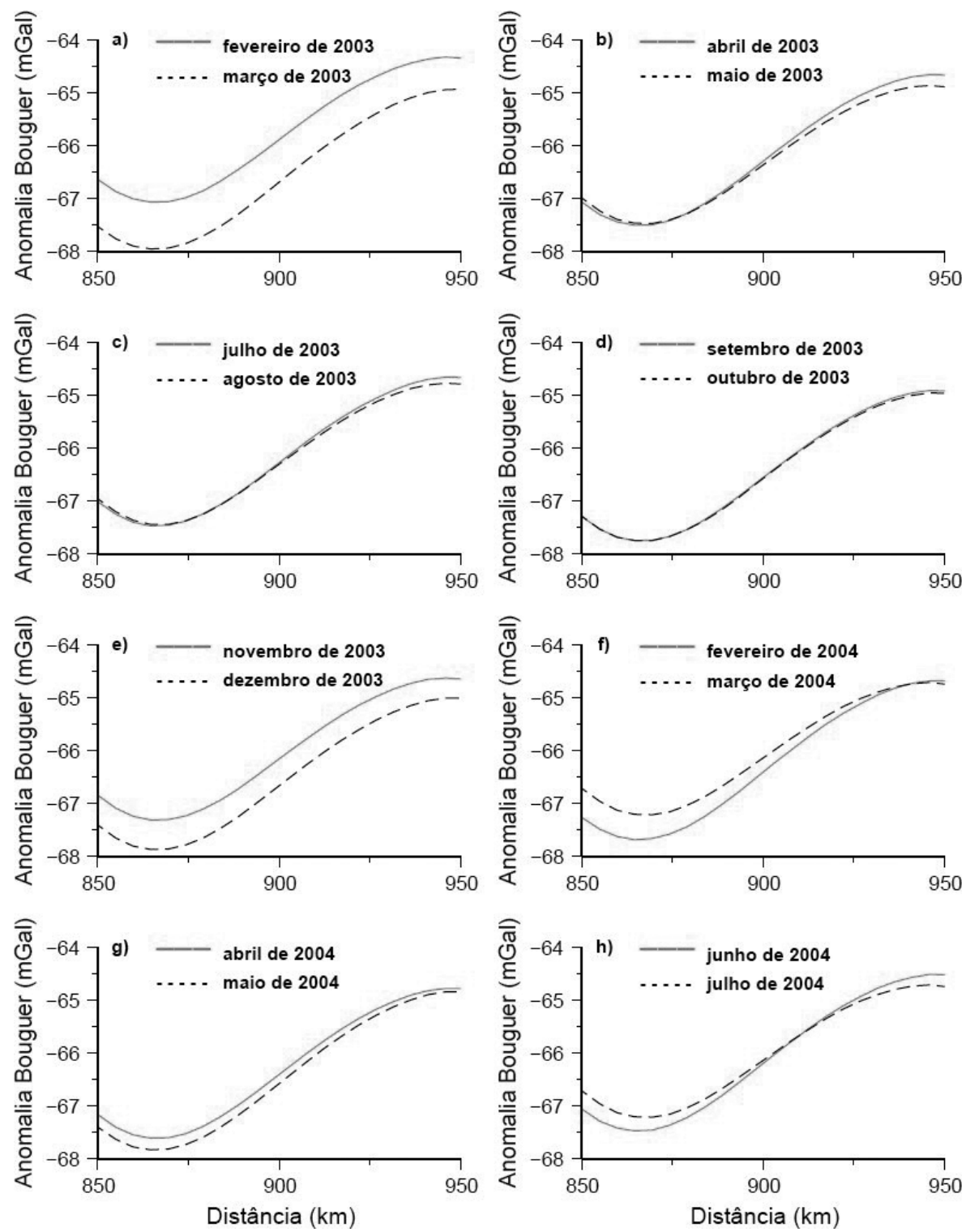

Figura 5 - Ampliação no trecho entre 850 e 950 km no Perfil A-A' da estimativa da anomalia Bouguer mês a mês.

consideradas provenientes das variações da massa de água subterrânea na região de estudo, a exemplo do estudo realizado por Wahr et al. (1998). A diferença entre o presente estudo e o citado é que aqui este procedimento é aplicado para as estimativas da anomalia Bouguer, e não para as diferenças das alturas geoidais, para se obter uma estimativa independente do efeito da variação das massas de água na anomalia gravimétrica.

\section{RESULTADOS}

Os resultados obtidos das diferenças das estimativas da anomalia Bouguer mostram um padrão ondulatório ao longo do perfil, que o torna um tanto difícil de interpretar geológica e geofisica- mente. Provavelmente este é o motivo que leva diversos pesquisadores a uma filtragem nos dados, como pode ser observado em Tapley (1997); Wahr et al. (1998); Rodell \& Famiglietti (1999, 2001 e 2002); Swenson \& Wahr (2002); Tapley et al. (2004); Wahr et al. (2004); Ramillien et al. (2004, 2005); Andersen \& Hinderer (2005); Chen et al. (2005); Gerrits (2005); Reigber et al. (2005). No presente trabalho tentou-se utilizar o mesmo conceito de análise com os dados de anomalia Bouguer ao longo de um perfil representativo da região estudada.

Na tentativa de avaliar a variação da massa de água que justificasse a anomalia Bouguer residual estimada a partir dos dados do GRACE, foram calculadas as médias das amplitudes pico-a- 


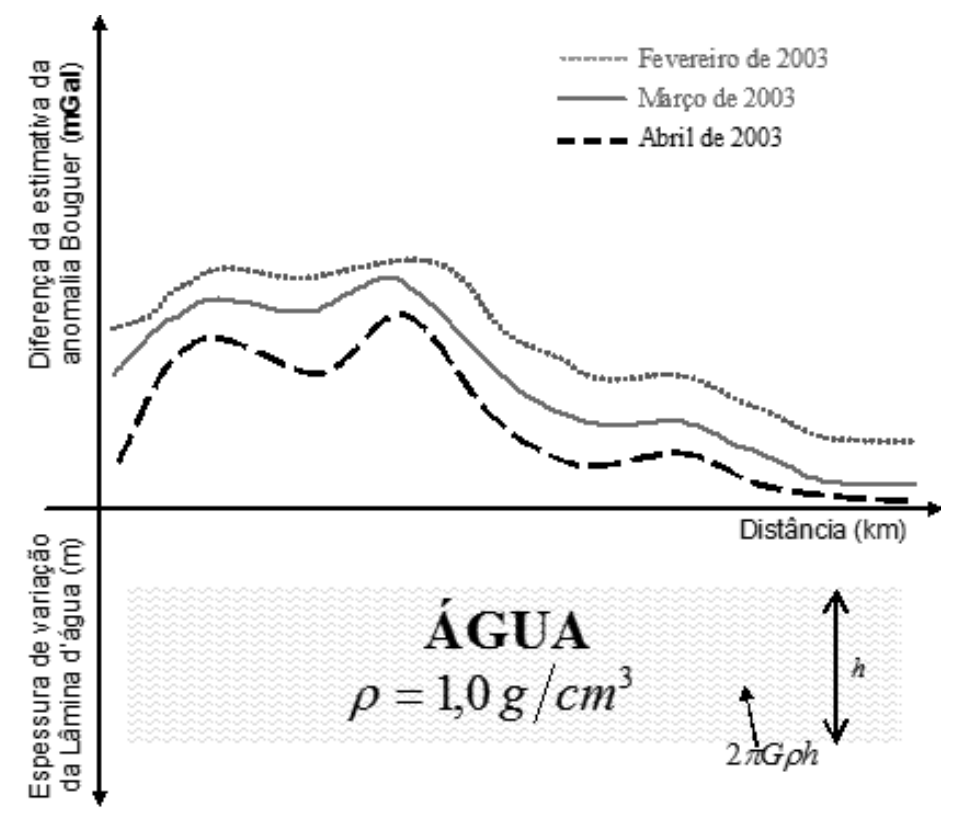

Figura 6 - Estimativa esquemática do volume de água necessário para obter as diferenças da estimativa da anomalia Bouguer mês a mês.

pico destas variações, que oscilam em torno de uma média zero, resultando em anomalias dadas na Figura 8.

Em março de 2003 obteve-se 0 maior valor para estimativa das diferenças pico-a-pico da anomalia Bouguer, 0 que indicaria uma maior variação do volume de água do aqüífero, considerando-se que as hipóteses assumidas neste trabalho são válidas. Por outro lado, em abril de 2002 observa-se 0 menor valor da variação da anomalia Bouguer, sugerindo que nesta ocasião houve a menor variação no volume de água do aqǘfero.

A Figura 9 mostra a variação das médias da anomalia Bouguer mês a mês para os dados investigados.

A partir da análise dos valores obtidos na Figura 9 foram calculados os valores de $h$ que representariam a variação de água na forma de um platô de Bouguer que justificaria a anomalia Bouguer residual média para cada solução mensal obtida para 0 período de abril de 2002 a julho de 2004 (Fig. 10). Nota-se que 0 valor médio da altura $h$ calculada fica em torno de $17 \mathrm{~m}$, com variação ao longo dos meses.

\section{Inversão linear 3-D das anomalias residuais do geóide}

As anomalias do geóide, entende-se aqui como alturas geoidais residuais mensais, pois são as diferenças (remoção) de uma altura geoidal média (estimada pelos valores das variáveis dos co- eficientes dos harmônicos esféricos $C_{l m}$ e $S_{l m}$ calculados a partir de um conjunto de dados abrangendo um período de coleta de aproximadamente 376 dias pelos satélites GRACE) em relação a cada altura geoidal mensal (estimada a partir de valores para as variáveis dos coeficientes dos harmônicos esféricos $C_{l m} \mathrm{e}$ $S_{l m}$ mensais, isto é, coeficientes são calculados a partir de um conjunto de dados abrangendo um período de coleta de aproximadamente 30 dias, aproximadamente um mês, pelos satélites GRACE), são importantes para caracterizar estruturas geológicas de grande porte de uma determinada região. Em particular, os dados do campo de gravidade da Terra fornecidos pela missão GRACE em coeficientes do geopotencial convertidos em alturas geoidais residuais na região de estudo (Sistema Aquúfero Guarani - SAG), após o processamento dos dados de cada solução mensal, poderão fornecer informações importantes relacionadas à variabilidade das massas de água subterrânea do SAG.

Neste trabalho, as modelagens direta e inversa foram realizadas com a metodologia de inversão linear 3-D de anomalias do geóide (Leite, 2005), que utiliza um funcional de inversão para minimizar parâmetros de corpos com variação de densidade a partir de anomalias do geóide, onde o funcional a ser resolvido relaciona o potencial da gravidade diretamente com os parâmetros físicos do meio. Wahr et al. (1998) já haviam ressaltado a possibilidade do uso das anomalias residuais do geóide no processo de inversão 3-D. 

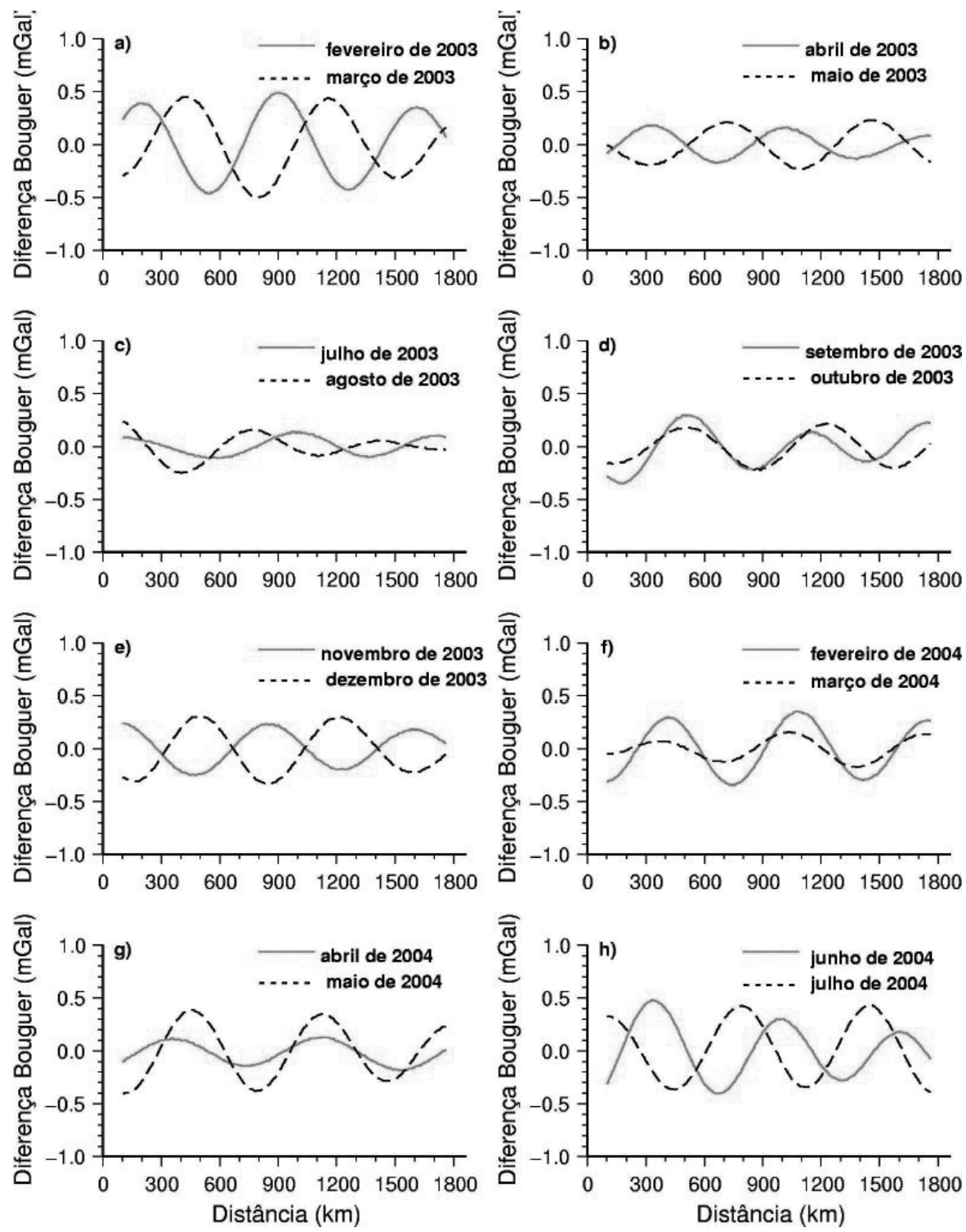

Figura 7 - Diferenças das estimativas da anomalia Bouguer mês a mês no Perfil A-A'

A Figura 11 representa o mapa geoidal da média de 376 dias de dados coletados pelos satélites GRACE com uma grade de 0.1 grau truncada em grau e ordem 70 , pois os erros para os graus dos coeficientes dos harmônicos esféricos maiores que 70 são grandes quando comparados aos valores da altura geoidal, mostrando a área quadrada (em vermelho) utilizada para a inversão das anomalias residuais do geóide, uma área de $6^{\circ} \times 6^{\circ}$ entre $20.5^{\circ}$ e $26.5^{\circ}$ de latitude sul e entre $48.5^{\circ}$ e $54.5^{\circ}$ de longitude oeste, que compreende a porção oeste do Estado do Mato Grosso do Sul, toda parte oeste do Estado de São Paulo e todo o Estado do Paraná.
Os parâmetros de entrada requeridos na inversão são: (a) mapa de anomalias residuais de geóide (a grade de dados das anomalias residuais de geóide); (b) incertezas nos dados de entrada; (c) configuração dos blocos usados no modelo da Terra; e (d) vínculos absolutos e relativos. Ao iniciar o procedimento, a matriz sensibilidade é calculada para um conjunto de dados e configuração de blocos no interior da Terra. Então $0 \Delta \rho$ (contraste de densidade) é calculado e $N$ (a altura geoidal mensal obtida dos dados do GRACE) é obtido. Por sua vez, $N$ é comparado com os dados de entrada de anomalia do geóide $\left(N^{\circ}\right)$, e o processo é reiniciado até se conseguir a convergência dos valores 

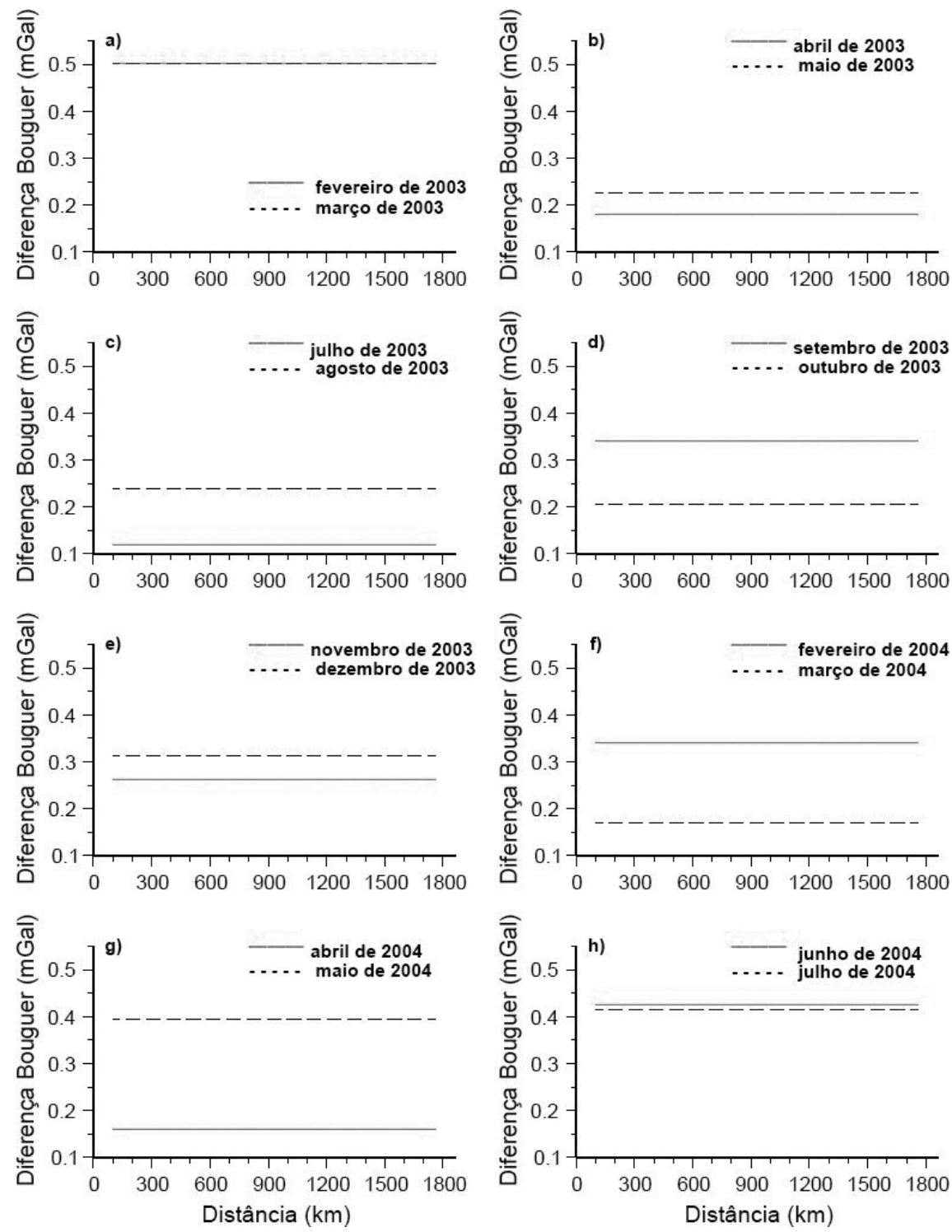

Figura 8 - Média das diferenças das estimativas da anomalia Bouguer mês a mês no Perfil A-A' obtida na Figura 7.

dentro de um grau de certeza pré-estabelecido. Uma descrição mais detalhada do procedimento de inversão linear 3-D de anomalias do geóide pode ser encontrada em Leite (2005).

Como dito anteriormente, a altura geoidal utilizada aqui é a diferença de um geóide mensal $(N)$ em relação a um geóide médio $(\bar{N})$, ambos obtidos dos coeficientes do geopotencial dos harmônicos esféricos, que é a forma disponível dos dados dos satélites GRACE à comunidade científica, portanto, foi utilizada uma mudança $(\Delta N)$, conforme a Eq. (3), para fazer a inversão.

A análise utilizada neste trabalho tem como premissa que os efeitos das formações sedimentares, do embasamento, dos sedimentos e das rochas ínneas são removidos dos dados iniciais, restando apenas a contribuição da variação das massas de água, que é a grandeza a ser estimada neste trabalho. A variação das anomalias do geóide residuais é atribuída à variação de água, de forma que toda a água será reduzida a uma camada, levando-se em consideração a diferença de densidade (contraste de densidade) entre a água e 0 ar, pela ausência de rochas e sedimentos (cujo efeito foi removido previamente). A espessura média do aqǘfero foi de 250 metros e a porosidade efetiva de $15 \%$, ou seja, um contraste de densidade de $0,15 \mathrm{~g} / \mathrm{cm}^{3}$.

Como a inversão realizada é linear, 0 algoritmo é 0 mesmo que foi utilizado no cálculo da matriz de sensibilidade, pois nesse caso cada valor da matriz é simplesmente o potencial gravitacio- 


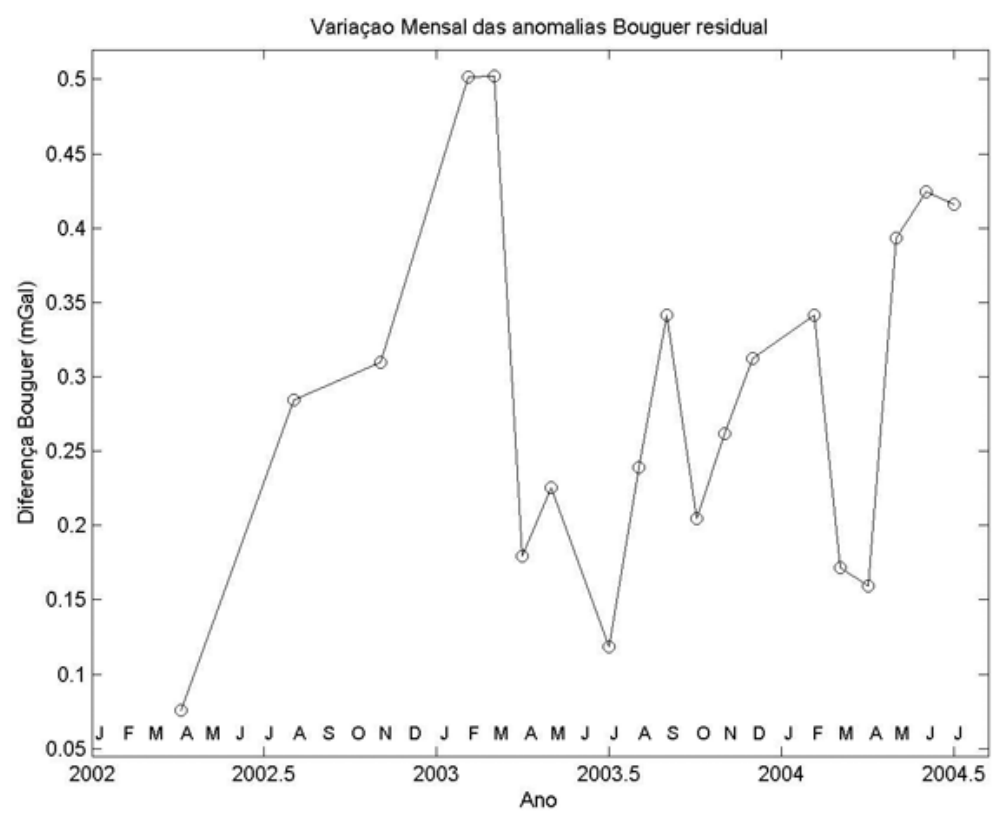

Figura 9 - Variação das estimativas médias das diferenças da anomalia Bouguer mês a mês no Perfil A-A'.

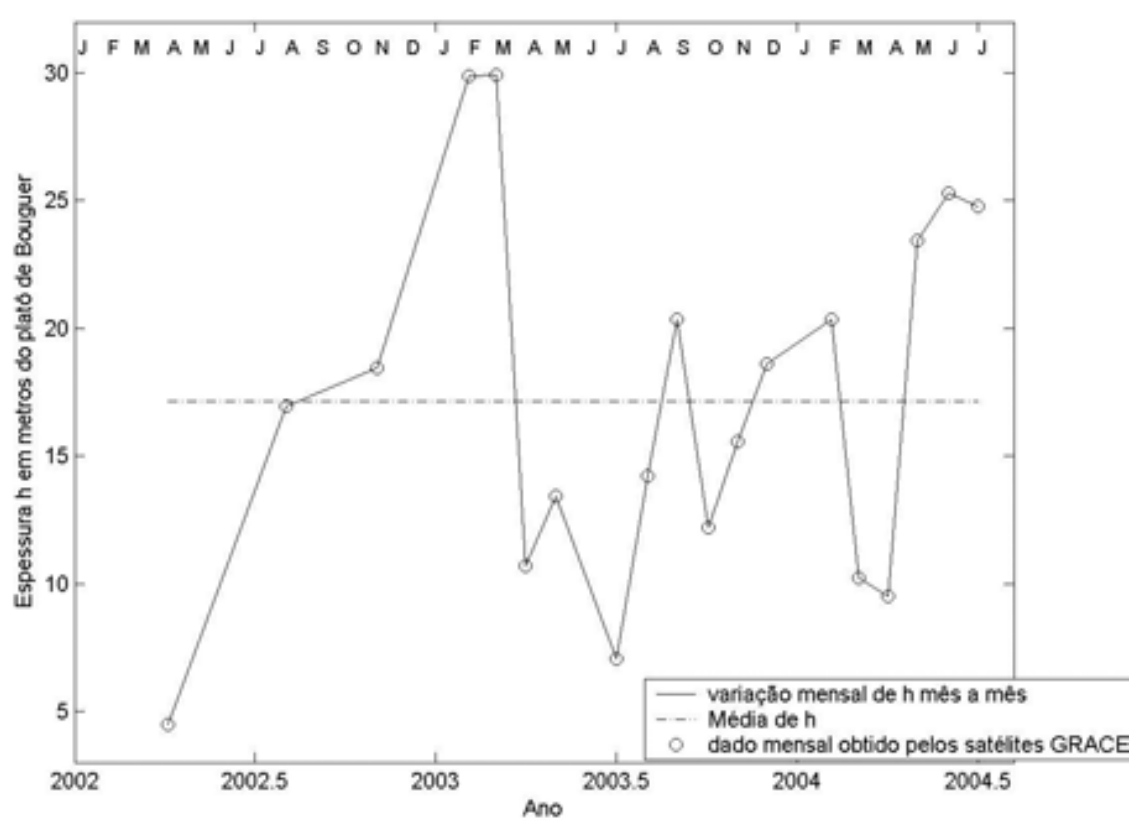

Figura 10 - Variação da espessura h do platô de Bouguer analisado nas anomalias Bouguer residuais médias no Perfil A-A'.

nal dos prismas nos pontos de observação, onde cada prisma tem um contraste de densidade unitário. 0 cálculo direto é importante, pois é necessária a comparação da anomalia residual do geóide calculado (causada pela distribuição de densidades) com a anomalia observada. A Figura 12 mostra a modelagem direta da anomalia residual do geóide de fevereiro de 2003 para uma camada de água do aquífero de $250 \mathrm{~m}$, necessária para a obtenção da matriz de sensibilidade utilizada na inversão dos dados calculados com a configuração dos blocos mostrada na Figura 13.

Para a anomalia residual do geóide observada, por exemplo, para fevereiro de 2003, deve haver uma fonte de contraste de densidade de forma a compatibilizar os valores calculados aos valores observados. A anomalia residual do geóide apresentada na 


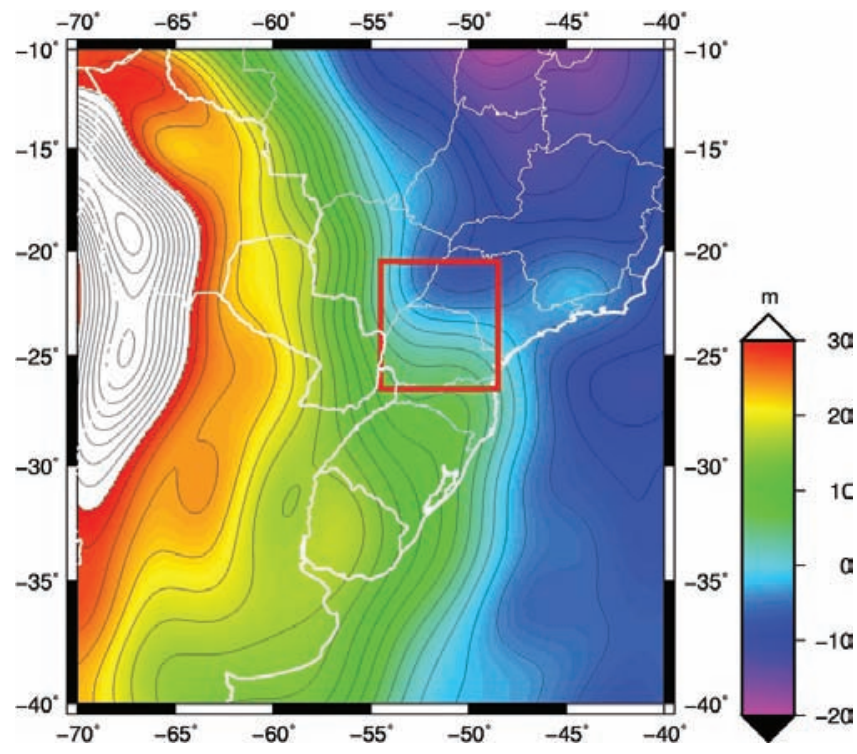

Figura 11 - Área de $6^{\circ} \times 6^{\circ}$ utilizada para inversão 3-D dos dados da altura geoidal residual (das diferenças geoidais).
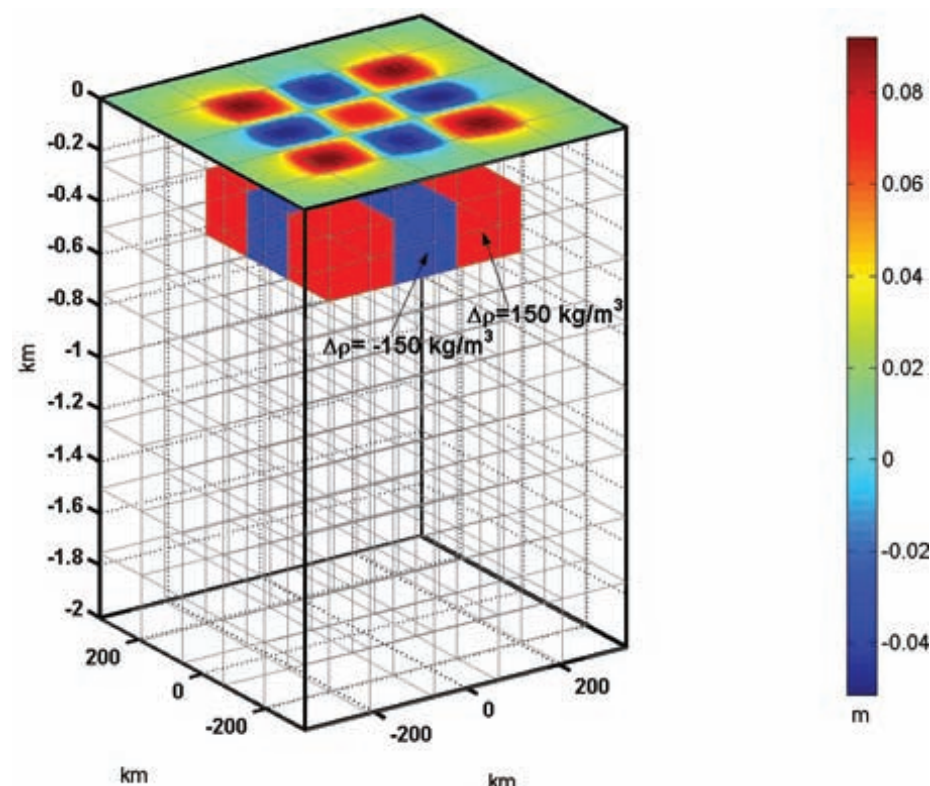

$\mathrm{km}$

$\mathrm{km}$

Figura 12 - Anomalia residual do geóide gerada pela combinação dos efeitos de cada prisma (blocos vermelhos e azuis) e seus respectivos contrastes de densidades obtidos para gerar a matriz de sensibilidade a ser utilizada na inversão da Figura 16. Estes prismas situam-se entre 250 e $500 \mathrm{~m}$ de profundidade.

Figura 12 é intercalada por fontes de densidade menor que o meio circundante e maior que o meio circundante situado entre $250 \mathrm{e}$ 500 metros de profundidade, conforme mostra o modelo de densidades com seu correspondente mapa de anomalias residuais do geóide calculado. Uma explicação possível para estes contrastes de densidade é a diferente porosidade efetiva das rochas e arenitos que formam 0 SAG, que explicaria as características dos dados obtidos do GRACE, que mostram este padrão intercalado nas anomalias residuais dos geóides obtidos.

0 procedimento de inversão foi então aplicado à anomalia residual do geóide da Figura 12, com 0 objetivo de checar se a distribuição de densidades obtida a partir da inversão reproduz a fonte da anomalia de densidade. 0 vínculo absoluto $\left(\mu_{a}\right)$ e 0 vínculo relativo $\left(\mu_{r}\right)$ que resultaram em modelos de densidades 
que representam melhor a fonte que produz a anomalia observada foram $\mu_{a}=1$ e $\mu_{r}=0,001$. Para inversão foi utilizado os limites laterais da grade de dados.

No exemplo apresentado para o geóide residual de fevereiro de 2003 é utilizado a configuração apresentada na Figura 13. Para fins de obter resultados de inversão satisfatórios com o modelo da Figura 13, a camada 1, os primeiros $250 \mathrm{~m}$ do modelo, teve valores fixados em $\Delta \rho=0 \mathrm{~kg} / \mathrm{m}^{3}$, representando os $250 \mathrm{~m}$ iniciais da bacia do Paraná que ficam acima do Aqüífero Guarani, cujo efeito foi removido com o cálculo da anomalia do geóide residual; portanto foi considerado $0 \Delta \rho=0 \mathrm{~kg} / \mathrm{m}^{3}$ e 0 valor de $\Delta \rho$ da camada $\mathrm{B}$ como relativo às rochas saturadas com água (arenitos e fissuras em basaltos) que apresentam uma porosidade média efetiva de $15 \%$, ou seja, apresenta um contraste de densidade de $-150 \mathrm{~kg} / \mathrm{m}^{3}$ e $/$ ou $150 \mathrm{~kg} / \mathrm{m}^{3}$. No modelo da Figura 13 segue uma configuração de 8 camadas de $250 \mathrm{~m}$ de espessura; a camada 1 vai de 0 a $250 \mathrm{~m}$, a camada 2 vai de 250 a $500 \mathrm{~m}$, e assim sucessivamente até a camada 8 que vai $1750 \mathrm{~m}$ até $2000 \mathrm{~m}$.

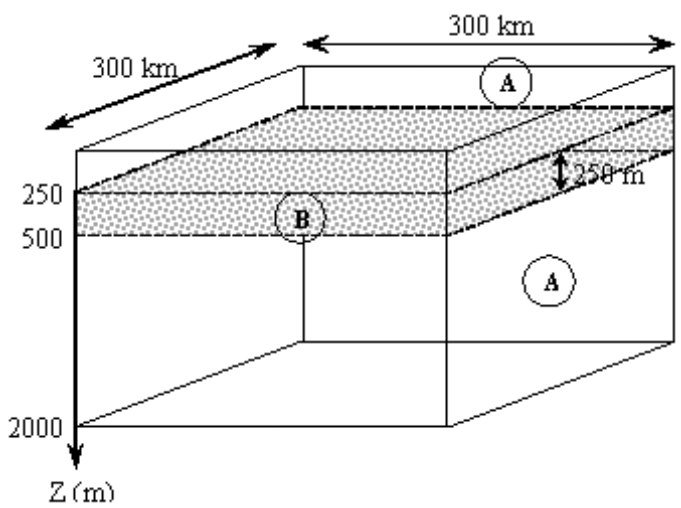

Figura 13 - Modelo de um aqǘfero, fora de escala, utilizado para gerar dados de entrada da inversão. A camada $A$ representa as formações sedimentares, embasamento, sedimentos e rochas ínneas de $250 \mathrm{~m}$ cujo efeito foi removido com 0 cálculo da anomalia do geóide residual e, portanto tiveram valores fixados em $\Delta \rho=0 \mathrm{~kg} / \mathrm{m}^{3}$. A camada $B$ representa uma camada saturada de água de 250 $\mathrm{m}$ de espessura com $\Delta \rho$ intercalado lateralmente entre os blocos com valores de $-150 \mathrm{~kg} / \mathrm{m}^{3}$ e $150 \mathrm{~kg} / \mathrm{m}^{3}$

A Figura 14 mostra os dados da missão GRACE, altura geoidal de fevereiro de 2003 utilizados para a inversão da Figura 17. 0 cálculo gerado na Figura 12 foi obter a matriz de sensibilidade a ser utilizada juntamente com os dados da Figura 14 para obter a inversão da Figura 17.

As Figuras 15 e 16 mostram as seções transversais do contraste de densidade compreendidas em um intervalo de zero a $2000 \mathrm{~m}$ de profundidade dividida em 8 camadas de $250 \mathrm{~m}$ de espessura por seção transversal obtidas a partir da configuração dos blocos apresentados na Figura 12.
A partir dos dados de entrada, modelo do campo gravitacional da Terra da missão GRACE para os meses que vão de fevereiro de 2003 a julho de 2004 fornecidos em forma de coeficientes dos harmônicos esféricos para grau e ordem 120 e 150 juntamente com os erros associados, foram processados e obtidos as respectivas alturas geoidal e anomalias ar-livre para cada conjunto destas soluções mensais onde resultou na inversão em forma de modelos de densidade das Figuras 15 e 16 das anomalias geoidais (alturas geoidais residuais). Por sua vez, os modelos de densidade foram utilizados para obter o modelo geoidal da Figura 17.

Observa-se que a primeira camada, que vai de 0 a $250 \mathrm{~m}$, apresenta valor zero para o contraste de densidade. Este resultado era esperado porque essa camada foi fixada previamente com valor nulo antes da realização da inversão, ou seja, assumese que a fonte anômala está situada abaixo de $250 \mathrm{~m}$ de profundidade. Se 0 resultado da inversão tivesse resolução e acurácia perfeitas, as células da camada 2 deveriam apresentar valores alternados respectivamente iguais à -150 e $150 \mathrm{~kg} / \mathrm{m}^{3}$, pois como já observado anteriormente, considera-se ter variação no volume de água apenas na camada 2, a qual é a camada B (de $250 \mathrm{~m}$ a $500 \mathrm{~m}$ ) da configuração do Aqüífero Guarani mostrada na Figura 13. Os valores das camadas 3, 4, 5, 6, 7 e 8 apresentam valores próximos de zero uma vez que estas camadas também foram fixadas previamente com valor nulo antes da realização da inversão. Nota-se que, pelos valores calculados pela inversão, é possível distinguir um corpo com contraste de densidade anômala positiva máxima de aproximadamente $75 \mathrm{~kg} / \mathrm{m}^{3}$. No entanto, o corpo "real" que está localizado entre 250 e $500 \mathrm{~m}$ tem contraste de densidade máxima de $150 \mathrm{~kg} / \mathrm{m}^{3}$ e mínima de $-150 \mathrm{~kg} / \mathrm{m}^{3}$. Nota-se também 0 contraste de densidade negativa decaindo até aproximadamente $-87 \mathrm{~kg} / \mathrm{m}^{3}$, o que corresponde ao corpo com densidade anômala negativa que alterna com 0 de densidade anômala positiva localizado também entre 250 e $500 \mathrm{~m}$.

Nesse caso, as soluções mostram suavidade, o que é característica de métodos lineares que utilizam fatores de estabilização.

\section{DISCUSSÃO E CONCLUSÕES}

A inversão linear 3-D das anomalias residuais do geóide foi obtida para a anomalia residual do geóide de fevereiro de 2003. Estas anomalias residuais são as alturas geoidais residuais da solução mensal de fevereiro de 2003 em relação a uma média de 376 dias de dados coletados pelos satélites GRACE.

A análise realizada neste trabalho mostrou que as anomalias residuais obtidas a partir dos coeficientes dos harmônicos 


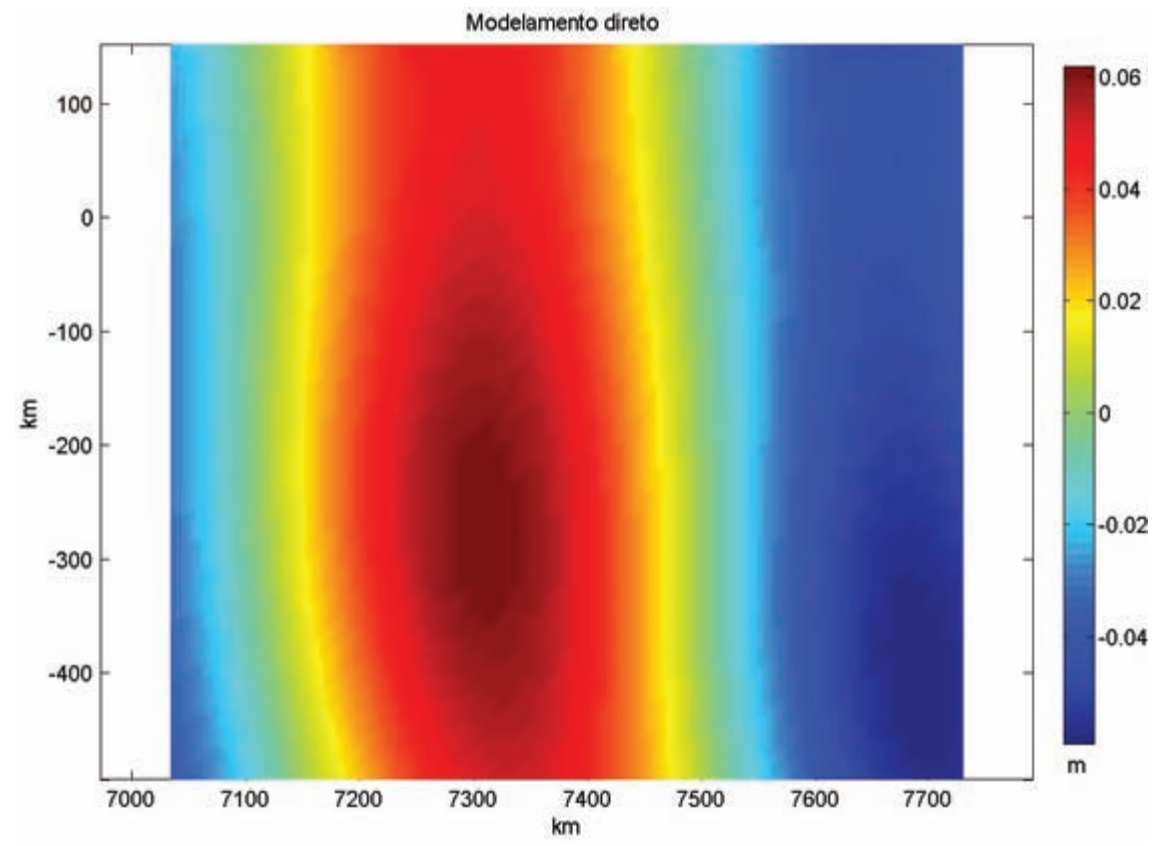

Figura 14 - Anomalia residual do geóide produzida pelo modelo geométrico apresentado na Figura 12.

(a) $z=250 \mathrm{~m}$

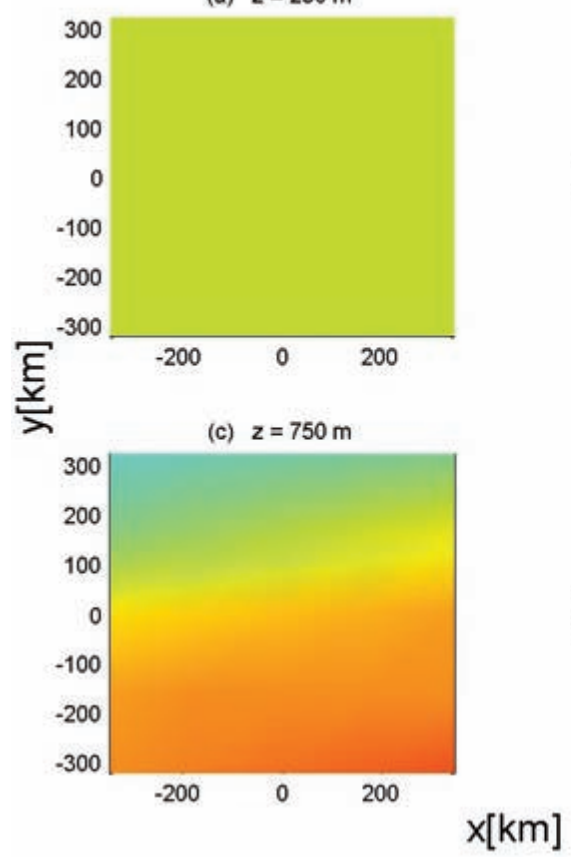

(b) $z=500 \mathrm{~m}$

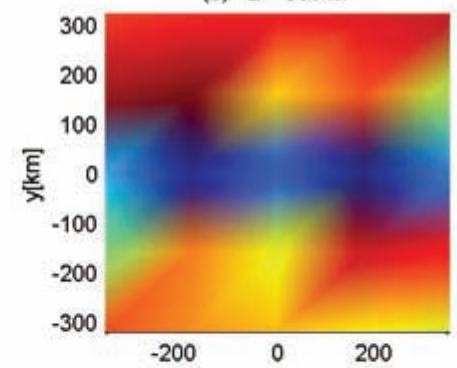

(d) $z=1000 \mathrm{~m}$

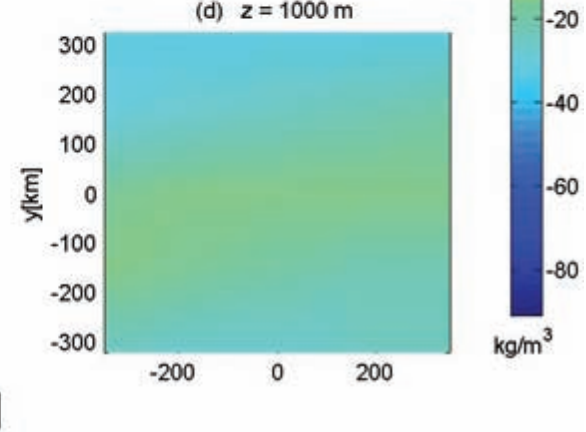

Figura 15 - Seções transversais de densidade, de $250 \mathrm{~m}$ de espessura cada, extraídas do modelo 3-D obtido a partir da configuração dos blocos apresentados na Figura 12, em profundidades: (a) 0-250 m; (b) 250-500 m; (c) 500-750 m; (d) 750-1000 m. A inversão foi realizada dentro da área limitada pelas coordenadas $x=(-347.8338 ;+347.8338) \mathrm{km}$, $y=(-322.5653 ;+322.5653) \mathrm{km}$ e $z=(0 ; 2) \mathrm{km}$. 
(a) $z=1250 \mathrm{~m}$

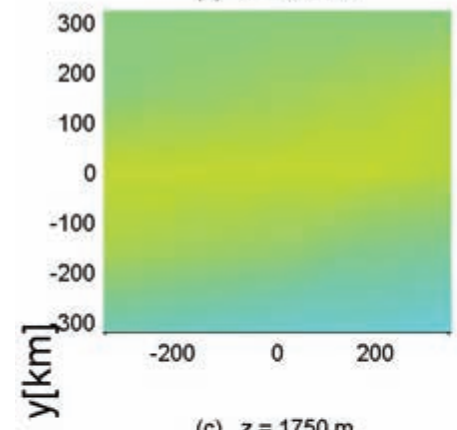

(c) $z=1750 \mathrm{~m}$

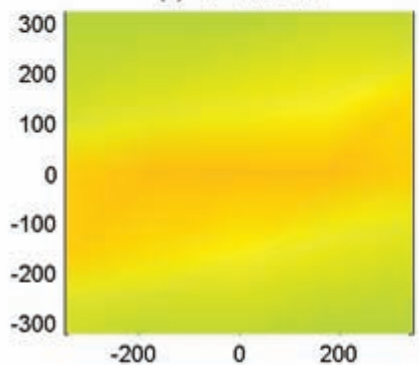

(b) $z=1500 \mathrm{~m}$

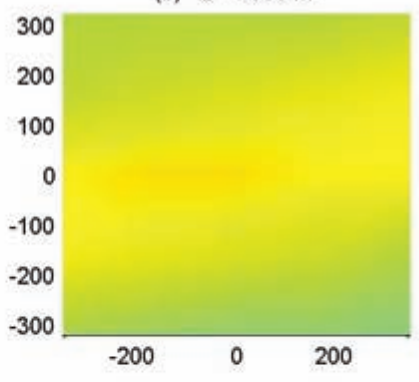

(d) $z=2000 \mathrm{~m}$

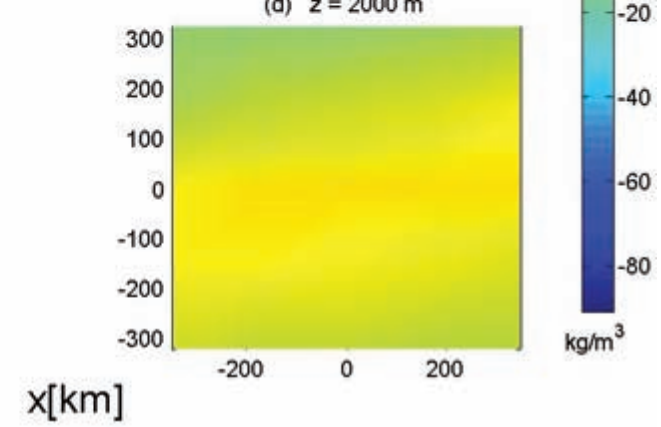

Figura 16 - Seções transversais de densidade, de 250 m de espessura cada, extraídas do modelo 3-D obtido a partir da configuração dos blocos apresentados na Figura 12, em profundidades: (a) 1000-1250 m; (b) 1250-1500 m; (c) 1500-1750 m; (d) 1750-2000 m. A inversão foi realizada dentro da área limitada pelas coordenadas $x=$ $(-347.8338 ;+347.8338) \mathrm{km}, y=(-322.5653 ;+322.5653) \mathrm{km} \mathrm{e} z=(0 ; 2) \mathrm{km}$.

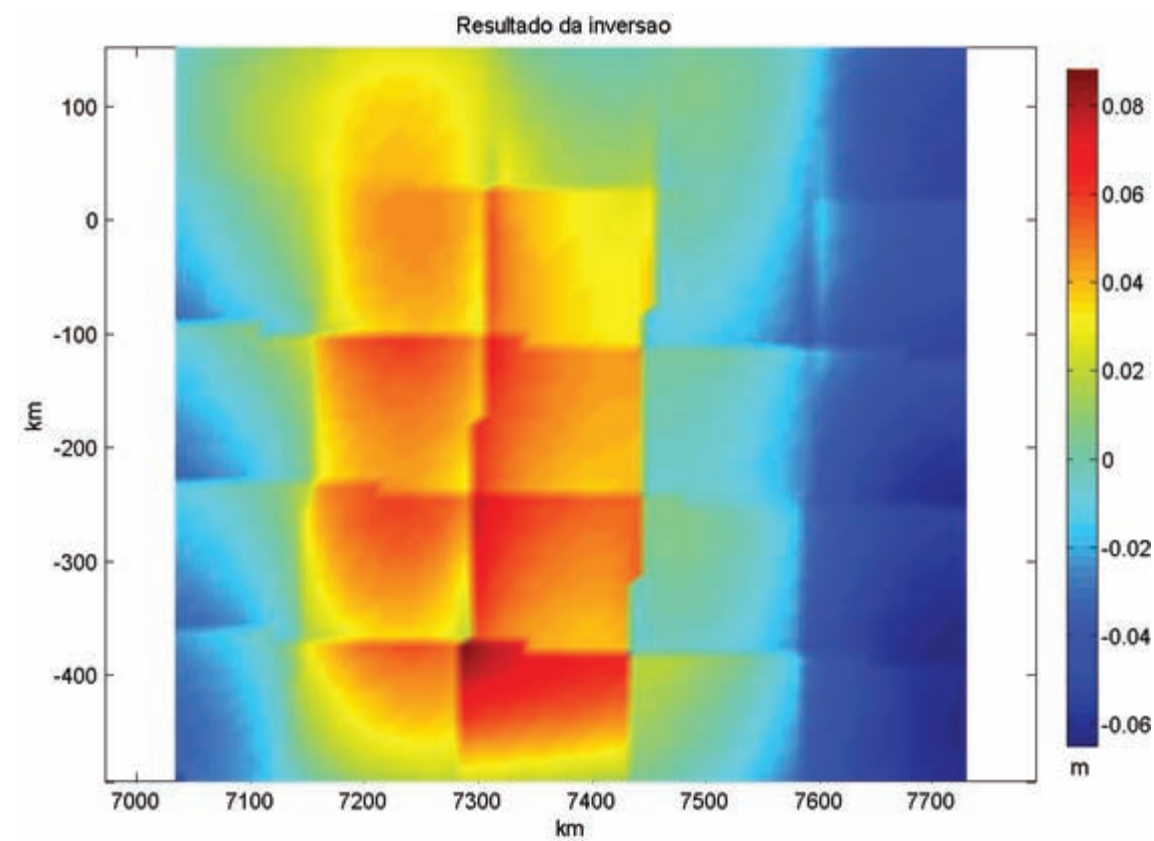

Figura 17 - Anomalia residual do geóide gerada a partir do modelo de densidades obtido com a inversão do mapa da Figura 16. 
esféricos obtidos dos satélites GRACE podem ser utilizadas para estimar a variação de massas de água em subsuperfície, pois a inversão 3-D permitiu recuperar os valores de anomalia observados com uma distribuição de densidades que poderia representar uma parte do Aqüiffero Guarani. A baixa resolução do modelo geoidal, Figura 17, calculado a partir dos modelos de densidade das Figuras 15 e 16, pode ser atribuída à falta de uma implementação adequada de um filtro que permita eliminar dos dados residuais da altura geoidal a componente gerada pela distribuição não uniforme dos dados, utilizando o método de Wahr et al. (1998), essa implementação poderia permitir apenas a contribuição da variação das massas de água em subsuperfície e está em fase de estudo.

A obtenção das anomalias Bouguer residuais foi utilizada como uma estimativa de mudanças no armazenamento das massas de água em termos das variações mensais de amplitudes das massas água, o que se caracteriza em uma técnica importante, uma vez que a previsão das mudanças do armazenamento de água é uma tarefa muito difícil, mesmo com modelos computacionais sofisticados. Além disso, a abordagem de se estimar variações de massas de águas a partir de dados do campo gravitacional variável no tempo (Warh et al., 1998), tais como, os dados obtidos dos satélites GRACE não é uma forma de medir quantidades exatas do armazenamento de água do espaço, e sim, estimar a mudança no armazenamento de água com o tempo. As estimativas realizadas mostram um maior valor da anomalia para fevereiro e março de 2003 e junho e julho de 2004, e os menores valores para abril de 2002, 2003 e 2004.

Ainda não foi possível realizar uma análise hidrológica ou de precipitação mensal para a região no mesmo período das análises obtidas pelos dados do GRACE, de forma que se pudesse verificar a relação dos dados de pluviosidade com os dados do GRACE. Como indicativo de que os dados de anomalia Bouguer provenientes do GRACE podem fornecer informação sobre a variação de massa de água no SAG, porém, foi realizado um teste para estimar qual a variação de massa de água que poderia gerar os valores observados de anomalia, sem fazer nenhuma hipótese sobre a geometria da distribuição de massa. Mesmo de maneira rudimentar, esta análise indica que 0 GRACE pode fornecer informações importantes para a modelagem hidrológica em grandes aqüíferos, como é o caso do Aqüífero Guarani.

\section{AGRADECIMENTOS}

Os autores agradecem à FAPESP (Fundação de Amparo à Pesquisa do Estado de São Paulo) e ao CNPq (Conselho Nacio- nal de Desenvolvimento Científico e Tecnológico - Processo 476171/2007-5 - Edital Universal 15/2007) pelo apoio financeiro no desenvolvimento desta pesquisa, a Emilson Pereira Leite pela disponibilidade do programa de inversão e pela ajuda na utilização do mesmo, ao Departamento de Geofísica do IAG/USP pelo uso de sua estrutura durante a realização desta pesquisa e aos dois revisores anônimos que contribuíram para 0 aprimoramento do manuscrito.

\section{REFERÊNCIAS}

ANDERSEN OB \& HINDERER J. 2005. Global inter-annual gravity changes from GRACE: Early results. Geophysical Research Letters, v. 32, L01402.

CETESB. 2001. Relatório de qualidade das águas subterrâneas no Estado de São Paulo 1998-2000 - São Paulo. 104pp. + anexos: il.; $30 \mathrm{~cm}$. (Série Relatórios CETESB, ISSN 0103-4103).

CETESB. 2004. Relatório de qualidade das águas subterrâneas no Estado de São Paulo 2001-2003 - São Paulo. 96pp. + anexos: il.; 30 cm. (Série Relatórios CETESB, ISSN 0103-4103).

CHEN JL, WILSON CR, FAMIGLIETTI JS \& RODELL M. 2005. Spatial sensitivity of the Gravity Recovery and Climate Experiment (GRACE) time-variable gravity observations. J. Geophys. Res., 110, B08408, doi:10.1029/ 2004JB003536.

GERRITS AMJ. 2005. Hydrological modelling of the Zambezi catchment for gravity measurements. MSc Thesis. TU Delft, The Netherlands. $184 \mathrm{pp}$.

HEISKANEN W \& MORITZ H. 1967. Physical Geodesy. W.H. Freeman and Company, San Francisco, 364 pp.

IAG. International Association of Geodesy. 1971. Geodetic reference system 1967. IAG Special Publication n. 3, Paris, France. 115 pp.

LEITE EP. 2005. Inversão 3-D de Anomalias do Geóide com Aplicação no Estudo da Evolução Térmica da Margem Continental Leste do Brasil. Tese de doutorado, USP, São Paulo, 185 pp.

RAMILLIEN G, CAZENAVE A \& BRUNAU 0. 2004. Global time variations of hydrological signals from GRACE satellite gravimetry. Geophysical Journal International, 158: 813-826.

RAMILLIEN G, FRAPPART F, CAZENAVE A \& GÜNTNER A. 2005. Time variations of land water storage from an inversion of two years of GRACE geoids. Earth and Planetary Science Letters, 235: 283-301.

REIGBER C, SCHMIDT R, FLECHTNER F, KÖNIG R, MEYER U, NEUMAYER KH, SCHWINTZER P \& ZHU SY. 2003. First GFZ GRACE Gravity Field Model EIGEN-GRACE01S. Disponível em: < http://op.gfzpotsdam.de/grace/results/grav/g001_eigen-grace01s.html>. Acesso em: 3 maio 2006. 
REIGBER C, SCHMIDT R, FLECHTNER F, KÖNIG R, MEYER U, NEUMAYER KH, SCHWINTZER P \& ZHU SY. 2005. An Earth gravity field model complete to degree and order 150 from GRACE: EIGENGRACE02S. Journal of Geodynamics, 39: 1-10.

ROCHA GA. 1997. 0 grande manancial do Cone Sul. Estudos Avançados, USP, 11(30): 191-212.

RODELL M \& FAMIGLIETTI JS. 1999. Detectability of variations in continental water storage from satellite observations of the time dependent gravity field. Water Resources Research, 35(9): 2705-2723.

RODELL M \& FAMILGLIETTI JS. 2001. An analysis of terrestrial water storage variations in Illinois with implications for the Gravity Recovery and Climate Experiment (GRACE). Water Resources Research, 37(5): 2705-2723.

RODELL M \& FAMIGLIETTI JS. 2002. The potential for satellite-based monitoring of groundwater storage changes using GRACE: the High Plains aquifer, Central US. Journal of Hydrology, 263: 245-256.

RODELL M, HOUSER PR, JAMBOR U, GOTTSCHALCK J, MITCHELL K, MENG CJ, ARSENAULT K, COSGROVE B, RADAKOVICH J, BOSILOVICH M, ENTIN JK, WALKER JP, LOHMANN D \& TOLL D. 2004. The Global Land Data Assimilation System, Bull. Amer. Meteor. Soc., 85: 381-394. SÁ NC, USSAMI N \& MOLINA EC. 1993. Gravity map of Brazil 1: representation of free-air and Bouguer anomalies. Journal of Geophysical
Research, Washington, 98(B2): 2187-2197.

SWENSON S \& WAHR J. 2002. Methods for inferring regional surfacemass anomalies from Gravity Recovery and Climate Experiment (GRACE) measurements of time-variable gravity. Journal of Geophysical Research, 107(B9): 2193.

TAPLEY BD. 1997. The gravity recovery and climate experiment (GRACE). American Geophysical Union, Fall Meeting. Eos Trans. AGU78 (48), F163

TAPLEY BD, BETTADPUR S, RIES JC, THOMPSON PF \& WATKINS MM. 2004. GRACE measurements of mass variability in the Earth System. Science, 305(5683): 503-505.

VAN ZYL JJ. 2001. The Shuttle Radar Topography Mission (SRTM): a breakthrough in remote sensing of topography. Acta Astronautica, 48: $559-565$.

WAHR J, MOLENAAR M \& BRYAN F. 1998. Time variability of the Earth's gravity field: Hydrological and oceanic effects and their possible detection using GRACE. Journal of Geophysical Research-Solid Earth, 103(B12): 30205-30229.

WAHR J, SWENSON S, ZLOTNICKI V \& VELICOGNA I. 2004. Timevariable gravity from GRACE: First results. Geophys. Res. Lett., 31, L11501, doi:10.1029/2004GL019779.

\section{NOTAS SOBRE OS AUTORES}

Everton Pereira Bomfim. Possui graduação em Física Bacharelado pela Universidade Estadual de Santa Cruz (2004) e mestrado em Geofísica pela Universidade de São Paulo (2006). Tem experiência na área de Física, com ênfase em Geofísica, atuando principalmente nos seguintes temas: gravimetria, campo de gravidade, harmônicos esféricos, gradiometria por satélite, geodésia física.

Eder Cassola Molina. Bacharel em Geofísica pelo Instituto de Astronomia, Geofísica e Ciências Atmosféricas - IAG/USP em 1987, mestre em Geofísica pelo IAG-USP em 1991, Doutor em Geofísica pelo IAG-USP em 1996. Atua na área de métodos potenciais como pesquisador e docente do Departamento de Geofísica do IAG-USP desde 1988 